\title{
Prevalence of allergic rhinitis comorbidity with asthma and asthma with allergic rhinitis in China: A meta-analysis
}

\author{
Yang Shen, ${ }^{1}$ Ji-Hong Zeng, ${ }^{1}$ Su-Ling Hong, ${ }^{1}$ Hou-Yong Kang ${ }^{1}$
}

\begin{abstract}
Background: Allergic rhinitis (AR) and asthma are the most common inflammatory diseases of the airways. The relationship between asthma and AR is widely and clinically recognised. The concept "one airway, one disease" has been gradually accepted. However, in China, we could not find any systematic review and meta-analysis on the prevalence of AR with asthma and asthma with AR.
\end{abstract}

Objective: The aim of this research was to carry out a meta-analysis on the results of all conducted studies to present valid information about the co-occurrence rate of AR with asthma and asthma with AR in China.

Methods: Pubmed/Medline, Science, Springer, Elsevier, Embase, Wanfang data, VIP, CBM, and CNKI were searched systemically and data were extracted from eligible studies by two independent reviewers. Meta-analysis, study quality assessment, and publication bias assessments were all done using Stata 12.1 software.

Results: The results of this meta-analysis showed that pooled prevalence estimates of AR with asthma ranged from $6.69 \%$ to $14.35 \%$, asthma with AR from $26.67 \%$ to $54 \%$. Furthermore, an overall prevalence of $10.17 \%$ (95\% CI 9.08-11.27\%) was ascertained for AR with asthma, and 38.97\% (95\% CI 34.42-43.53\%) for asthma with AR.

Conclusions: The present meta-analysis comprehensively provided the first quantitative summary of the prevalence of AR with asthma and asthma with AR in China. Our study demonstrated that, in China, asthma and AR are often comorbid diseases and co-exist in the same patients. There is a close correlation between AR and asthma from an epidemiological standpoint.

Key words: allergic rhinitis, asthma, comorbidity, prevalence, China

\section{From:}

${ }^{1}$ Department of Otorhinolaryngology, The First Affiliated Hospital of Chongqing Medical University, Chongqing, People's Republic of China

\section{Corresponding author:}

Hou-Yong Kang

Department of Otorhinolaryngology, The First Affiliated Hospital of Chongqing Medical University,

1\#Yixueyuan Road, Chongqing 400016, People's Republic of China E-mail: khy_cq@sina.com

The relationship between asthma and AR is widely and clinically recognised. Grossman first described the concept "one airway, one disease" in 1997, mainly from the pathophysiological roles of leukotriene inflammation in the upper and lower airways. ${ }^{6}$ Research showed that many patients with asthma, particularly those with allergic asthma, also have AR. The mucosa of the upper and lower airways is continuous, and the types of inflammation in AR and asthma are very similar, involving $\mathrm{T}$ helper type 2 cells, mast cells, and eosinophils. Both diseases have characteristic symptoms and are strongly 
influenced by environmental factors. Previous studies demonstrated that among patients with asthma and concomitant $\mathrm{AR}$, those who received treatment for AR had a significantly lower risk of subsequent asthma-related events (emergency care visits /hospitalisations) than those who did not receive treatment. ${ }^{7}$ Ohta et al. found that in Japan, AR is a common comorbidity $(67.3 \%)$ in asthma and that it impairs asthma control. ${ }^{8}$

The data about the prevalence of allergic rhinitis, asthma among the Chinese population may affect the decision of policy makers, insurance organisations, and health authorities. Although, there are a few studies about the prevalence of AR and asthma in China, we could not find any systematic review and meta-analysis on the prevalence of asthma and AR among the Chinese population, especially the prevalence of AR with asthma and asthma with AR. Thus, the aim of this research was to carry out a meta-analysis on the results of all conducted studies to present valid information about the prevalence of AR with asthma. In addition, we aimed to investigate the co -occurrence rate of AR with asthma and asthma with AR in China.

\section{Materials and Methods}

Preferred reporting items for systematic reviews and meta -analyses (PRISMA) guidelines were followed while performing this meta-analysis and associated systematic review. ${ }^{9}$

\section{Literature search}

Sensitive, systematic searches were separately conducted by two trained researchers to find studies on allergic rhinitis and asthma. Several electronic databases including Pubmed/ Medline, Science, Springer, Elsevier, Embase, Wanfang data, VIP, CBM, and CNKI were searched for relevant articles. The major medical subject headings $(\mathrm{MeSH})$ and keywords used in different logical combinations and phrases included "allergic rhinitis", "asthma”, "epidemiology/prevalence/morbidity/incidence/attack rate", and "comorbidity". The search encompassed original research papers published from 2006 to 2016.

\section{Inclusion and exclusion criteria}

We included population-based studies that reported the prevalence of allergic rhinitis and asthma among Chinese populations. The inclusion criteria were: (1) studies reporting the prevalence of allergic rhinitis, asthma, allergic rhinitis with asthma, and/or asthma with allergic rhinitis; (2) studies reporting the exact diagnostic criteria; (3) cross-sectional studies; and (4) study reports with data in forms that were able to be utilised in the meta-analysis. The exclusion criteria were: (1) repeated publications; (2) reviews; (3) studies providing insufficient data; and (4) a methodological quality score less than 5 .

\section{Data extraction}

Initially, two researchers independently reviewed all the titles and abstracts that were selected using the keywords. In the second phase, full texts of the articles, which were selected in the first phase, were reviewed; finally, the researchers selected the articles whose contents were suitable for data extraction. Disagreements between the two reviewers about selecting articles were resolved by a third reviewer via discussion and consensus. Extracted information included name of the first author, year of publication, type of study (local study or survey), total sample size, number of patients, point prevalence, and $95 \%$ confidence interval (CI) of point prevalence.

\section{Study quality assessment}

The global burden of disease quality assessment checklist was used to assess the quality of the studies. Total study quality score was achieved by summing the sampling method (1-4 score), the sample size $(0-3)$, and the response rate $(0-6) .{ }^{10}$

\section{Statistical analysis}

The AR with asthma and asthma with AR prevalences were calculated using the random effects model with 95\% CI. To evaluate heterogeneity, we estimated the proportion of between-study inconsistency using the $\mathrm{I}^{2}$ statistic, with values of $25 \%, 50 \%$, and $75 \%$ considered low, moderate, and high, respectively. If the heterogeneity was significant and $\mathrm{I}^{2}>50 \%$, the random-effect model was adopted; otherwise, the fixed -effect model was used. All statistical tests were performed using Stata software version 12.1 (Stata Corporation, College Station, TX, USA).

\section{Results \\ Literature search}

Following the development of our search strategy, a total of 783 relevant articles were selected from primary research in electronic databases. After deleting duplicate articles and reviews, 325 potential articles were obtained. Then, 278 articles were excluded due to irrelevance to the study subject after evaluation of titles and abstracts, so 47 articles were included into the study for reviewing full-text. Finally, 26 articles were excluded after reviewing full-texts due to inappropriate study design and/or outcome. Thus, 21 studies that met inclusion criteria were included in the meta-analysis and summarised in Figure 1 and Table. 1.

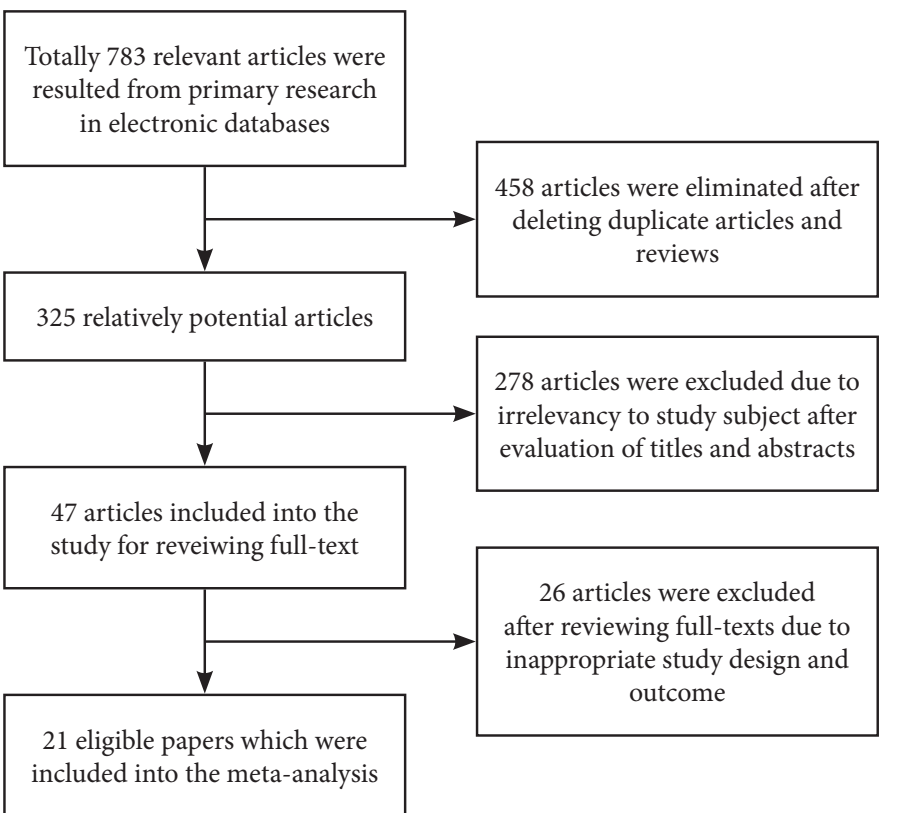

Figure 1. Flowchart for identification of studies selected. 
Table 1. Characteristics of the included studies on prevalence of AR with asthma.

\begin{tabular}{|c|c|c|c|c|c|c|c|c|}
\hline \multicolumn{9}{|c|}{ AR with asthma } \\
\hline Year & Author & Study & Age (y) & Diagnosis & AR & Asthma & Sample & Rate \\
\hline 2015 & Gao Rongli & Cross-sectional study & $5-70$ & ARIA & 248 & 20 & 2052 & $8.06 \%$ \\
\hline 2015 & Zhang Liangran & Cross-sectional study & $5-80$ & ISAAC & 690 & 76 & 2778 & $11.01 \%$ \\
\hline 2015 & Yang Li & Cross-sectional study & $2-81$ & ARIA & 324 & 22 & 8716 & $6.79 \%$ \\
\hline 2015 & Chen Xing & Cross-sectional study & $18-70$ & ARIA & 425 & 61 & 2580 & $14.35 \%$ \\
\hline 2014 & Liu Xiaoling & Cross-sectional study & $5-66$ & ARIA & 266 & 19 & 266 & $7.14 \%$ \\
\hline 2014 & Wang Wenya & Cross-sectional study & $\geq 14$ & ARIA & 3859 & 355 & 3859 & $9.20 \%$ \\
\hline 2012 & Fu Jingming & Cross-sectional study & $7-75$ & ARIA & 164 & 20 & 916 & $12.20 \%$ \\
\hline 2011 & Zhu Xiuqing & Cross-sectional study & $7-75$ & ARIA & 672 & 78 & 2516 & $11.61 \%$ \\
\hline 2010 & Yin Rong & Cross-sectional study & $2-81$ & ARIA & 2267 & 238 & 2267 & $10.50 \%$ \\
\hline 2009 & Dou Xiuli & Cross-sectional study & $>15$ & ISAAC & 901 & 101 & 6026 & $11.21 \%$ \\
\hline 2008 & Yin Haihong & Cross-sectional study & $18-24$ & ISAAC & 226 & 26 & 1954 & $11.50 \%$ \\
\hline \multicolumn{9}{|c|}{ Asthma with AR } \\
\hline Year & Author & Study & Age (y) & Diagnosis & Asthma & AR & Sample & Rate \\
\hline 2015 & Li Jipeng & Cross-sectional study & $\geq 4$ & ARIA & 174 & 79 & 14412 & $45.40 \%$ \\
\hline 2015 & Feng Qiuyue & Cross-sectional study & $0-99$ & ARIA & 45 & 12 & 20000 & $26.67 \%$ \\
\hline 2014 & Pan Huiming & Cross-sectional study & $16-82$ & ARIA & 212 & 78 & 212 & 36.79 \\
\hline 2014 & Li Jiaowu & Cross-sectional study & $7-92$ & ARIA & 72 & 21 & 6909 & $29.17 \%$ \\
\hline 2013 & Wang Wenya & Cross-sectional study & $>14$ & ARIA & 687 & 226 & 57647 & $32.90 \%$ \\
\hline 2013 & Li Seng & Cross-sectional study & $12-78$ & ARIA & 300 & 162 & 300 & $54.00 \%$ \\
\hline 2011 & Qian Juanjuan & Cross-sectional study & $\geq 4$ & ARIA & 95 & 43 & 4956 & $45.26 \%$ \\
\hline 2010 & $\mathrm{Ma} \mathrm{Li}$ & Cross-sectional study & $0-85$ & ISAAC & 731 & 296 & 731 & $40.49 \%$ \\
\hline 2009 & Zhou Lin & Cross-sectional study & $>15$ & ARIA & 73 & 23 & 5216 & $31.51 \%$ \\
\hline 2007 & Yu Qihong & Cross-sectional study & $14-82$ & ARIA & 793 & 316 & 793 & $37.85 \%$ \\
\hline
\end{tabular}
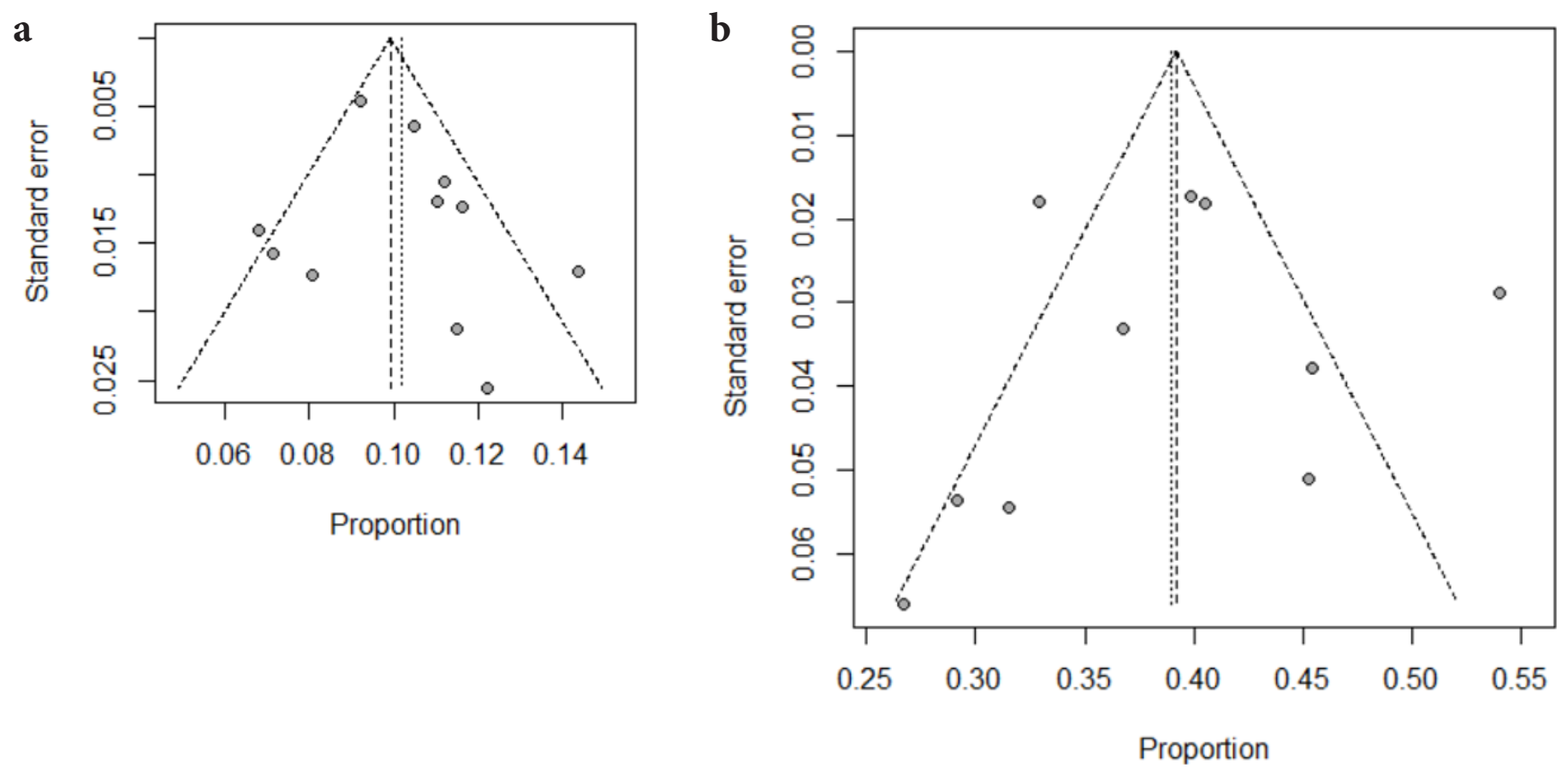

Figure 2. A funnel plot of the overall meta-analysis of metabolic and endocrine comorbidities reflecting publication bias. (a. AR with asthma; $b$. asthma with AR) 


\section{Study characteristics}

The selected studies were published from 2006 to 2016 and all the included articles were carried out as cross-sectional surveys, including 133813 participants and 10042 AR patients and 3182 asthma patients in the articles that comprised this meta-analysis. Publication bias assessment was made by visual examination of the funnel plot symmetry. (Figure 2)

\section{Estimated prevalence of AR comorbid with asthma}

Eleven studies ${ }^{11-21}$ about AR with asthma in China were selected in this research. Based on the results of random effect method, the overall prevalence of AR cmorbid with asthma in China was 10.17\% (95\% CI 9.08-11.27\%). In total, 10042 AR patients with an average of $913 \mathrm{AR}$ patients per study were evaluated. The highest prevalence was reported by Chen Xing et al. in 2015 (14.35\%) and the lowest by Yang Li et al. in 2015 (6.79\%). (Figure 3, Table 1)

\section{Estimated prevalence of asthma comorbid with AR}

Ten studies ${ }^{22-31}$ about asthma with AR in China were selected. The overall prevalence of asthma comorbid with AR in China was $38.97 \%$ (95\% CI 34.42-43.53\%). In total, 3182 asthma patients with an average of 32 asthma patients per study were evaluated. The highest prevalence was reported by $\mathrm{Li}$ Seng et al. in 2013 (54\%) and the lowest by Feng Qiuyue et al. in 2015 (26.67\%). (Figure 4, Table 1)

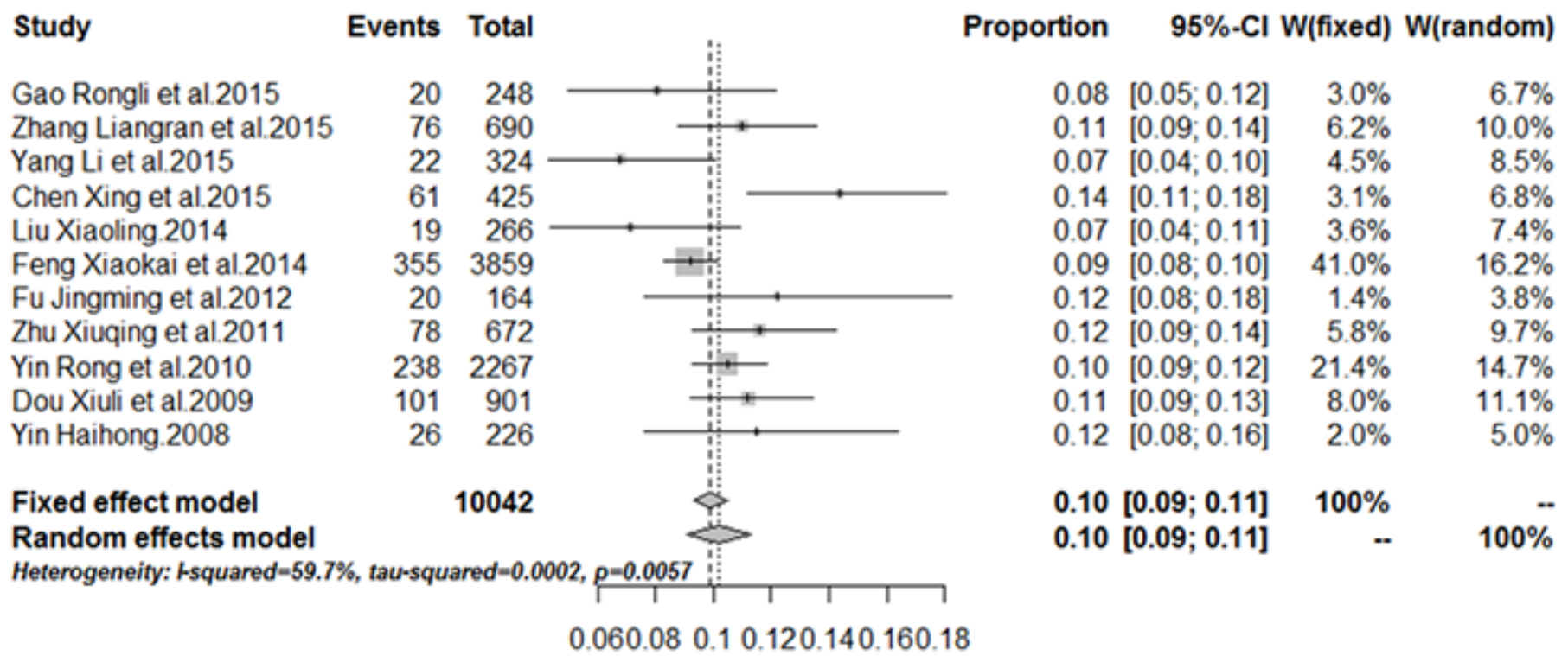

Figure 3. Forest plot of the rate of AR patients with asthma.

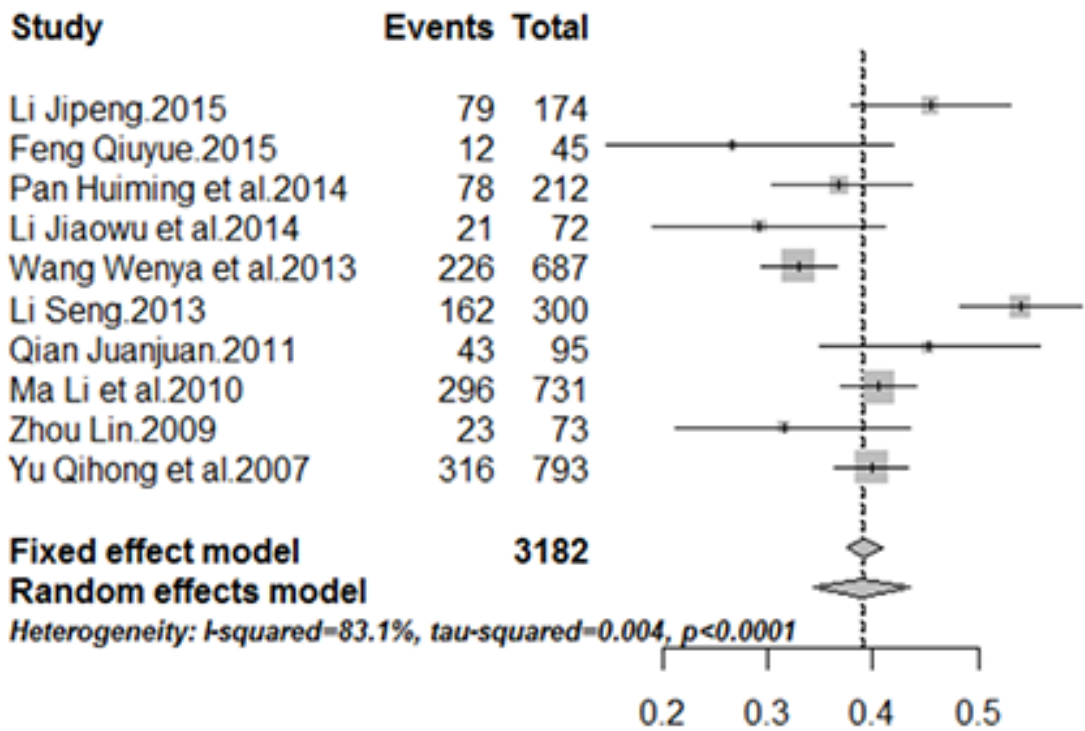

Figure 4. Forest plot of the rate of asthma patients with AR.
Proportion $\quad 95 \%-\mathrm{Cl}$ W(fixed) W(random)

$\begin{array}{rrrr}0.45[0.38 ; 0.53] & 5.2 \% & 10.0 \% \\ 0.27[0.15 ; 0.42] & 1.7 \% & 6.5 \% \\ 0.37[0.30 ; 0.44] & 6.7 \% & 10.6 \% \\ 0.29[0.19 ; 0.41] & 2.6 \% & 7.9 \% \\ 0.33[0.29 ; 0.37] & 22.9 \% & 12.6 \% \\ 0.54[0.48 ; 0.60] & 8.9 \% & 11.2 \% \\ 0.45[0.35 ; 0.56] & 2.8 \% & 8.2 \% \\ 0.40[0.37 ; 0.44] & 22.3 \% & 12.5 \% \\ 0.32[0.21 ; 0.43] & 2.5 \% & 7.8 \% \\ 0.40[0.36 ; 0.43] & 24.4 \% & 12.6 \% \\ & & & \\ \mathbf{0 . 3 9}[\mathbf{0 . 3 8} ; 0.41] & \mathbf{1 0 0 \%} & - \\ \mathbf{0 . 3 9}[0.34 ; 0.44] & -- & \mathbf{1 0 0 \%}\end{array}$




\section{Discussion}

Allergic rhinitis and asthma are both caused by an inappropriate immunological response to antigens compared to the response elicited in most individuals. Our study presented a comprehensive report about the prevalence of AR with asthma and asthma with AR. The results of this meta-analysis showed that pooled prevalence estimates of AR with asthma ranged from $6.69 \%$ to $14.35 \%$ and asthma with AR from $26.67 \%$ to $54 \%$. Furthermore, an overall prevalence of $10.17 \%$ (95\% CI 9.08-11.27\%) was determined for AR with asthma, and 38.97\% (95\% CI 34.42-43.53\%) for asthma with AR. This study presented a comprehensive report that is the first quantitative summary of the prevalence of AR with asthma and asthma with $\mathrm{AR}$ in China. The results of this meta-analysis demonstrated a close correlation between AR and asthma from an epidemiological perspective.

AR and asthma, rather than being considered two distinct diseases, can be unified by the concept of a "united airway," where allergic symptoms of the upper and lower airways can be thought of as manifestations of a common atopic entity. ${ }^{6,32}$ Both diseases, which are IgE mediated, can be triggered by similar allergens, including mold, animal dander, and house-dust mites. Epidemiological studies have shown that the majority of patients with asthma have concomitant rhinitis and the presence of rhinitis is an increased risk factor for the development of asthma. ${ }^{33,34}$ The prevalence of asthma is $<2 \%$ in subjects without rhinitis while it varies from $10 \%$ to $40 \%$ in patients with rhinitis. ${ }^{35}$ Meanwhile, AR occurs in $>75 \%$ of patients with asthma, whereas asthma affects up to $40 \%$ of patients with $\mathrm{AR}^{36}$ In a 10 -year longitudinal study of children with AR, asthma was eventually found in $19 \%$ of the cases, and in $25 \%$ of the sample size asthma and AR developed simultaneously. ${ }^{37}$ In a 23-year follow-up study of almost 2000 college students, patients with AR, when compared with controls without AR, were about three times more likely to develop asthma. ${ }^{38}$ Pefura-Yone et al. reported that the prevalence of rhinitis was $27.3 \%$ among subjects with current wheezing and $25.4 \%$ of participants with asthma had rhinitis in Cameroon. ${ }^{39}$ Furthermore, in Japan, a nationwide survey of asthmatic patients revealed that $67.3 \%$ of asthmatic patients had $A R .{ }^{8}$ In addition to the epidemiological evidence, several clinical reports point to a common pathophysiological relationship between $\mathrm{AR}$ and asthma. ${ }^{40}$ Our meta-analysis demonstrated the prevalence of AR with asthma and asthma with AR in China. The results supported that asthma and AR are often comorbid diseases and co-exist in the same patients. Meanwhile, our data showed the prevalence of asthmatic patients with AR in China to be lower than in Japan. On the one hand, we think the difference may partly be ascribed to regional disparity. On the other hand, environmental factors and different allergens may aos play roles. ${ }^{41}$

Based on the results of previous research and our meta -analysis, we know that there is a close correlation between $\mathrm{AR}$ and asthma; AR is highly comorbid with asthma and is a risk factor for asthma. These studies indicate that establishing the overall concept of upper and lower airway is particularly important for AR and asthma treatment. Thus, on the one hand, we should pay attention to the evaluation of the lower airway of AR patients, using pulmonary function tests, bronchial provocation experiment, chest radiograph, and so on. On the other hand, in the process of asthma treatment, we should note to control the symptoms of AR.

Nevertheless, there are some several limitations to the present meta-analysis. First, the number of studies included was comparatively small. Second, the lack of detailed descriptions of AR and asthma features (such as atopic status, age of onset, and disease severity) constrained further subgroup analyses. Third, our study only included the studies from the last 10 years. As we all know, the environment has changed greatly during this time span. Thus, the changes in environmental risk factors for AR may have partially biased the results of this meta-analysis. Meanwhile, in this research, only published studies were reviewed; as a result, unpublished studies and gray literature were not included in our analyses because they were not accessible. Such sets of data could have greatly impacted our results.

In conclusion, the present meta-analysis comprehensively provided the first quantitative summary of the prevalence of AR with asthma and asthma with AR in China. The results of this study showed that the overall prevalence of AR with asthma and asthma with AR was $10.17 \%$ and $38.97 \%$, respectively. Our study demonstrated that asthma and AR are often comorbid diseases and co-exist in the same patients. There is a close correlation between AR and asthma from an epidemiological perspective. These results can fill the knowledge gaps about the prevalence of respiratory diseases in China, and it can help policy makers, specialists, insurance companies, and all stockholders to make plans and evaluate the medical services required to reduce the prevalence of respiratory diseases.

\section{Disclosure statement}

The authors declare no financial or other conflicts of interest regarding the content of this article.

\section{Acknowledgments}

This study was supported by the National Natural Science Foundation of China (Grant No.81500774 and 81470676).

\section{References}

1. Björkstén B, Clayton T, Ellwood P, Stewart A, Strachan D; ISAAC Phase III Study Group. Worldwide time trends for symptoms of rhinitis and conjunctivitis: phase III of the International Study of Asthma and Allergies in Childhood. Pediatr Allergy Immunol. 2008;19(2):110-24.

2. Shen J, Ke X, Hong S, Zeng Q, Liang C, Li T, et al. Epidemiological features of allergic rhinitis in four major cities in Western China. J Huazhong Univ Sci Technolog Med Sci. 2011;31(4):433-40.

3. Gessner BD, Neeno T. Trends in asthma prevalence, hospitalization risk, and inhaled corticosteroid use among Alaska native and nonnative medicaid recipients younger than 20 years. Ann Allerg Asthma Im. 2005; 94(3):372-9.

4. Demoly P, Paggiaro P, Plaza V, Bolge S, Kannan H, Sohier B, et al. Prevalence of asthma control among adults in France, Germany, Italy, Spain and the UK. Eur Respir Rev. 2009; 18(112):105-12.

5. Musafiri S, van Meerbeeck J, Musango L, Brusselle G, Joos G, Seminega B, et al. Prevalence of atopy, asthma and COPD in an urban and a rural area of an African country. Respir Med. 2011; 105(11):1596-605.

6. Grossman J. One airway, one disease. Chest. 1997;111 Suppl 2:S11-6.

7. Corren J, Manning BE, Thompson SF, Hennessy S, Strom BL. Rhinitis therapy and the prevention of hospital care for asthma: a case-control study. J Allergy Clin Immunol. 2004;113(3):415-9. 
8. Ohta K, Bousquet PJ, Aizawa H, Akiyama K, Adachi M, Ichinose M, et al. Prevalence and impact of rhinitis in asthma. SACRA, a cross-sectional nation-wide study in Japan. Allergy. 2011;66(10):1287-95.

9. Moher D, Liberati A, Tetzlaff J, Altman DG; PRISMA Group. Preferred reporting items for systematic reviews and meta-analyses: the PRISMA Statement. Int J Surg. 2010;8(5):336-41.

10. Farzadfar F, Delavari A, Malekzadeh R, Mesdaghinia A, Jamshidi HR, Sayyari A, et al. NASBOD 2013: design, definitions, and metrics. Arch Iran Med. 2014; 17(1):7-15.

11. Gao RL, Ding J, Zang YW, Yan S, Liu TT, Liu ZG, et al. Epidemiological investigation of allergic rhinitis patients with asthma in Qingdao area. Progress in Modern Biomedicine. 2012; 12(15): 2891-95. Chinese.

12. Zhang LR, Epidemiological investigation and study of allergic rhinitis in the urban area of Kunming [dissertation]. [Kunming(KM)]: Kunming Medical College; 2015. 64 p.

13. Yang L, Shi DZ, Huang YJ. Investigation of allergic rhinitis in both rural and urban areas of hengyang city. Medical Innovation of China. 2015; 12(22):067-9. Chinese.

14. Chen X, Ji YY, Cheng P, Song CB, Yan JH, Zhu HL. Epidemiological investigation of allergic rhinitis in Ningbo area. Modern Practical Medicine. 2016; 28(3):377-9. Chinese.

15. Liu XL, Sun XL, Weng ZP, Liu SY. Clinical characters of the allergic rhinitis in Hohhot city. Chinese Archives of Otolaryngology-Head and Neck Surgery. 2013; 20(9):481-5. Chinese.

16. Wang WY, Lin JT, Su N, Liu GL, Feng XK, He QY, et al. Survey on the prevalence rate of bronchial asthma in Beijing area among the residents aged over 14 years from 2010 to 2011. Zhonghua Yi Xue Za Zhi. 2013; 93(18):1383-7. Chinese.

17. Fu JM. Xining area of allergic rhinitis sick and related factors analysis [dissertation]. Qinghai (QH): Qinghai College. 2012. 38 p.

18. Zhu XQ, Jang BF, Shi GG. Epidemiological investigation and analysis of allergic rhinitis in Luxi area. Shandong Medicine. 2009;49(29):67-8. Chinese.

19. Yin R, Liu SX, Liang CY, Hong SL. Survey on the epidemiological features of allergic rhinitis at out-patient in western area of China. Chinese Archives of Otolaryngology-Head and Neck Surgery. 2010;17(1):11-4. Chinese.

20. Dou XL. Epidemiologic investigation of risk factors of bronchial asthma in city proper of Qingdao [dissertation]. Taishang(TS): Taishang medical College. 2009. $51 \mathrm{p}$

21. Yin HY. Nanning city college of allergic rhinitis epidemiological investigation [dissertation]. Guangxi (GX): Guangxi University of Chinese Medicine. 2008. 35 p.

22. Li JP. Epidemiology survey and risk factors of bronchial asthma in Kunming. Journal of Clinical Pulmonary Medicine. 2015; 20(9):1667-9. Chinese.

23. Feng QY. Epidemiological survey and analysis on bronchial asthma in Huairou area. Capital Food And Medicine. 2015; 4: 24-6. Chinese.

24. Pan HM, Yan DM, Yao X, Liao SC, Chen TS. The clinical analysis of 212 cases of allergic rhinitis and bronchial asthma, Journal of Taishan Medical College. 2014; 35(4):284-6. Chinese.

25. Li JW, Huang JY, Guo FM, Fang J. Epidemiological studies of asthma complicated with allergic rhinitis. Chinese and Foreign Medical Research. 2014; 12(24):50-1. Chinese.
26. Wang WY. An epidemiology survey on the prevalence and associated risk factors of asthma among the residents who aged more than 14 years in Beijing from 2010 to 2011 [dissertation]. Peking (PK): Peking Union Medical College. 2013. 103 p.

27. Li S, Kong LF. A questionnaire survey of allergic rhinitis in bronchial asthma patients. Chinese Journal of Practical Internal Medicine. 2009; 29(12): 1139-40. Chinese.

28. Qian JJ, Ma JY, Zhou M, Zhou X. A survey on epidemiology and risk factors of bronchial asthma in Baoshan district of Shanghai. Journal of Internal Medicine Concepts \& Practice. 2011; 6(2):121-4. Chinese.

29. Ma L, Chen DL, Zhang RX, Wang XL, Shi YJ, Ji C, et al. A related heredity epidemiological research on allergic rhinitis and asthma in Nantong region. Chinese Journal of Otorhinolaryngology Head and Neck Surgery. 2010; 45(6):502-5. Chinese.

30. Zhou N, Cao J, Chen BY, Zhu BY, Deng Y. Lung function analysis and epidemiological survey of patients with bronchial asthma combined with allergic rhinitis in Tianjin area. Chinese Journal of Asthma. 2012;6(6): 425-8. Chinese.

31. Yu QY, Yang WJ, Lin YP. Report on epidemiological sampling survey of bronchial asthma in Tianjin area. Chinese Medical Association Fifth National Asthma Academic Conference and the first meeting of China Asthma Alliance; 2006 Aug 25-28; Changsha, Chinese. Hunan: 2007. p. 173.

32. Pawankar R, Bunnag C, Chen Y, Fukuda T, Kim YY, Le LT, Huong le TT, O’Hehir RE, Ohta K, Vichyanond P, Wang DY, Zhong N, Khaltaev $\mathrm{N}$, Bousquet J. Allergic rhinitis and its impact on asthma update (ARIA 2008)--western and Asian-Pacific perspective. Asian Pac J Allergy Immunol. 2009 Dec;27(4):237-43.

33. Khan DA. Allergic rhinitis and asthma: epidemiology and common pathophysiology. Allergy Asthma Proc. 2014;35(5):357-61.

34. Sritipsukho P, Satdhabudha A, Nanthapisal S. Effect of allergic rhinitis and asthma on the quality of life in young Thai adolescents. Asian Pac J Allergy Immunol. 2015 Sep;33(3):222-6.

35. Ozdoganoglu T, Songu M. The burden of allergic rhinitis and asthma.Ther Adv Respir Dis. 2012;6(1):11-23.

36. Bousquet J, Van Cauwenberge P, Khaltaev N; Aria Workshop Group; World Health Organization. Allergic rhinitis and its impact on asthma. J Allergy Clin Immunol. 2001;108(5 Suppl):S147-334

37. Settipane RJ, Hagy GW, and Settipane GA. Long-term risk factors for developing asthma and allergic rhinitis: A 23-year follow-up study of college students. Allergy Proc. 1994;15(1):21-5.

38. Huovinen E, Kaprio J, Laitinen LA, and Koskenvuo M. Incidence and prevalence of asthma among adult Finnish men and women of the Finnish Twin Cohort from 1975 to 1990 , and their relation to hay fever and chronic bronchitis. Chest. 1999;115(4):928-36.

39. Pefura-Yone EW, Kengne AP, Balkissou AD, Boulleys-Nana JR, Efe-de-Melingui NR, Ndjeutcheu-Moualeu PI, et al. Prevalence of asthma and allergic rhinitis among adults in Yaounde, Cameroon.PLoS One. 2015;10(4):e0123099.

40. Ciprandi G, Cirillo I, Tosca MA, and Vizzaccaro A. Bronchial hyperreactivity and spirometric impairment in patients with perennial allergic rhinitis. Int Arch Allergy Immunol. 2004;133(1):14-8.

41. Tham EH, Lee AJ1, Bever HV. Aeroallergen sensitization and allergic disease phenotypes in Asia. Asian Pac J Allergy Immunol. 2016 Sep; 34(3):181-189. 